\title{
Un modelo analítico para la intervención social: integrando los enfoques de las capacidades, el capital social y las instituciones en el ámbito local
}

\author{
Integrating perspectives on skills, social capital \\ and local institutions. An analitycal model for social \\ intervention
}

RenÉ Olate ${ }^{1}$

\begin{abstract}
Resumen
En este documento se entrega una aproximación a un modelo analítico de intervención social que integra los conceptos de capacidades, capital social e instituciones sociales. El modelo se articula en las realidades locales de América Latina y se destacan las implicancias que tiene para el avance sostenido de los procesos de desarrollo e inclusión social. Además, se entregan algunas sugerencias metodológicas para la intervención social.

Palabras claves: modelo analitico de intervención - capital social- procesos de desarrollo-inclusión social

Abstract

This document describes an approach to the analytical model of social intervention that integrates the concepts of skills, social capital and social institutions. The model is articulated in the local realities of Latin America and its implications for the sustained advance of the development processes and social inclusion is showed. Also some methodological suggestions for the social intervention are given.
\end{abstract}

Key words: analytical model of intervention - social capital - processes of social developmentinclusion"

Los marineros se convertirán en piratas si la sociedad crea incentivos para la piratería y los piratas se convertirán en comerciantes cuando la sociedad cree incentivos para el comercio" (Douglass North)

"Construir capital social no será fácil, pero es la clave para hacer funcionar la democracia" (Robert Putnam)

"Para resolver los problemas a los que nos enfrentamos, hemos de concebir la libertad individual como un compromiso social" (Amartya Sen)

1 Agradezco las sugerencias de Carlos Comas (Universidad Centroamericana de Nicaragua) así como los comentarios de los participantes en el seminario del Center for New Institucional Social Sciences que dirige el profesor Douglass North en Washington University in St. Louis. (c)Doctor en Trabajo Social Washington University in St. Louis rolate@wustl.edu 


\section{Introducción}

En las perspectivas teóricas sobre desarrollo se ha observado en las ultimas décadas un desplazamiento desde aquellas posiciones que enfatizaban la acumulación de capital como elemento central para lograr el desarrollo, hacia posiciones que primero enfatizaron la tecnología, luego el papel de las ideas y finalmente la importancia de las capacidades, el capital social y las instituciones.

Este desplazamiento en la concepción de desarrollo está marcado por el creciente reconocimiento de que las diferencias en resultados económicos no pueden ser completamente explicadas por diferencias en recursos tradicionales como la tierra, el trabajo y el capital físico. Al respecto, Hoff y Stiglitz (2001) señalan que el desarrollo ya no es visto como un proceso primario de acumulación de capital sino como un proceso de cambio organizacional. Este cambio no significa que la concepción tradicional de capital deje de ser importante en los procesos de desarrollo, sino que básicamente debe entenderse como otro factor dentro de una complejidad de elementos.

Dentro de esta complejidad se destacan tres concepciones. La concepción de las capacidades y del desarrollo como expansión de libertades elaborada por Amartya Sen y Martha Nussbaum.

La valoración de las relaciones sociales y del capital social como elemento explicativo de procesos de desarrollo económico y social elaborada originalmente, entre otros autores, por Pierre Bourdieu, James Coleman y Robert Putnam. Finalmente, la renovada importancia del tema de las instituciones sociales para explicar el desempeño de las economías y la sostenibilidad de los procesos de desarrollo, teoría desarrollada por diversos cientistas sociales entre los cuales se destaca el historiador económico Douglass North.

Un elemento coincidente en los tres conceptos, desde la disciplina de la economía es que todos surgen como reacción y crítica a la escuela neoclásica y a la teoría de la elección racional. A pesar de este origen común, y aceptando las diferencias al interior de cada corriente, esta tendencia al distanciamiento estaría más cercana a la complementariedad. Otro elemento común que es preciso destacar es el importante sendero de interdisciplinariedad que abren estos enfoques en los temas de desarrollo y de estrategias tendientes a combatir la pobreza y a lograr la inclusión social. Es difícil encontrar un planteamiento actual de desarrollo que no incluya a alguno de los elementos que consideran estos enfoques.
Más allá de las coincidencias en el plano teórico, este documento pretende plantear algunas preguntas relevantes en torno a las múltiples interrelaciones y mixturas que estos temas presentan en las realidades locales de América Latina, destacando la importancia que tienen para producir avances sostenidos en los procesos de desarrollo. Por tanto, el objetivo de este documento es presentar una primera aproximación a un modelo analítico de intervención social que integre los conceptos de capacidades, capital social e instituciones.

El argumento básico es que el desarrollo y la construcción de capacidades son procesos que se dan en el ámbito individual y que se pueden potenciar o limitar de acuerdo a las relaciones sociales o capital social, entendiendo a este último elemento en un contexto institucional. Analíticamente estos tres conceptos se pueden asociar a tres distintos niveles de análisis que se interrelacionan. Las capacidades se enfocan desde una perspectiva individual-grupal, el capital social desde una perspectiva grupal-comunitaria, y las instituciones desde una perspectiva comunitaria-societal. Se desprende como elemento central que para incentivar procesos de desarrollo desde la intervención social debemos considerar este enfoque que integra simultáneamente la construcción de capacidades y capital social, y la identificación, modificación o creación de instituciones sociales. Se deja planteada la necesidad de contrastar esta primera aproximación analítica a realidades concretas de América Latina, para de este modo poder profundizar su potencia explicativa y desarrollar sugerencias metodológicas para la intervención social.

El documento se estructura en cuatro partes. Las tres primeras están dedicadas a la presentación de cada perspectiva conceptual, señalando algunos resultados de investigaciones empíricas relevantes. La cuarta parte articula las tres perspectivas en el modelo analítico de capacidades, capital social e instituciones, sugiriendo algunos elementos explicativos y de intervención social. Finalmente, se entregan algunas dimensiones que habrá que seguir analizando para formular sugerencias metodológicas para la intervención social a nivel local.

\section{El enfoque de las capacidades}

El enfoque de las capacidades tiene como principales exponentes al premio Nóbel de Economía Amartya Sen y a la filósofa Martha Nussbaum. Nussbaum $(1995,2001)$ ha enfatizado los temas éticos y de género, destacando la idea de que las capa- 
cidades pueden servir como principios legales y constitucionales. Estos principios pueden ser utilizados por los ciudadanos para apelar ante el Estado. Sen $(1989,1999)$, por su parte, propone el enfoque de las capacidades ${ }^{2}$ basado en un análisis formal de los temas fundacionales de la renovación de la economía del bienestar, el análisis práctico de los problemas de pobreza, especialmente el problema de la hambruna, y una revisión de su planteamiento de las necesidades humanas básicas.

En esta revisión, Sen propone el concepto de titularidades, que hace referencia al modo que tienen las personas de acceder a los distintos bienes y servicios que satisfacen necesidades básicas. Sen entiende el concepto de titularidades como derechos económicos, distinguiendo entre titularidades de producción y titularidades de cambio.

Sin embargo, Sen ha sido criticado por su enfoque de las titularidades. Específicamente se considera que: a) no ha especificado cuáles son las necesidades básicas; b) no ha señalado si estas son iguales para todo el mundo y en cualquier tiempo; c) no ha señalado cómo se pueden justificar y qué es lo que determina una titularidad u otra (Pressman y Summerfield, 2000). Intentando responder a sus críticos, Sen centra sus estudios en lo que tiene un valor intrínseco para la vida, en las potencialidades más que en los bienes, en lo que entraña un valor instrumental o beneficio personal. A partir de este desarrollo propone el concepto de capacidades.

Sen (1999) define las capacidades como todo aquello que una persona es capaz de ser o hacer: estar bien nutrido, escribir, leer, comunicarse, tomar parte en la vida comunitaria, participar en actividades sociales, tener sentido de pertenencia a grupos. La capacidad es un tipo de libertad, la libertad fundamental de lograr distintos estilos de vida. Es conveniente señalar la distinción, que proviene de la traducción del inglés al español de los conceptos de capability y capacity. Ambos conceptos han sido traducidos al español como capacidad o capacidades. Sin embargo, aluden a vertientes conceptuales distintas.
La concepción de capacidades o capabilities de Sen alude a la idea de las potencialidades de los individuos. Es por ello que una clave para entender este concepto es cuando Sen sugiere que el núcleo del desarrollo consiste en expandir las capacidades de los individuos o sus potencialidades. En cambio, la idea de capacidades siguiendo el concepto de capacities se concentra en el ámbito organizacional o institucional. Por esta razón es frecuente encontrar en la literatura la idea de generación de capacidades organizacionales o institucionales. Por ejemplo, Moore (1995) menciona la generación de capacidades operativas en las organizaciones públicas y Repetto (2004) las capacidades estatales.

En el concepto de Sen, las necesidades básicas son entendidas dentro de las capacidades. Sen señala que la ausencia de capacidades conduce a las privaciones humanas. De este modo estructura su trabajo de las capacidades humanas y privaciones en torno a la importancia de ampliar las libertades, entendidas como la libertad de expresión, la libertad de prensa, la libertad para participar en las discusiones públicas, y de acceso a un sistema judicial transparente. Esta preocupación por las capacidades humanas y privaciones ha quedado plasmada en la construcción de los indicadores de desarrollo humano que utiliza Naciones Unidas.

En el contexto de las titularidades y las capacidades, Sen destaca la importancia de las instituciones. De este modo señala que las tradiciones, las leyes y las diferentes transferencias de bienestar pueden aumentar o disminuir las titularidades y el desarrollo o expansión de las capacidades. Por ello, es posible afirmar que tanto en los aspectos teóricos como en los prácticos, esta perspectiva señala importantes implicancias para los procesos de cambio institucional $^{3}$.

Sen afirma que los tipos de capacidades son muy variados, por ejemplo, desde tener acceso a un adecuado nivel de alimentación y nutrición hasta la participación en la vida comunitaria. Por ello señala que la expansión de las capacidades de las personas depende de la eliminación de las privaciones y de la

2 Amartya Sen ha planteado esta perspectiva en "Resources, Values and Development" (1984) y en "The Concept of Development" (1995) en H. Chenery y T. N. Srinavasan (eds.), Handbook of Development Economics. Los requisitos y las implicaciones más amplias de esta perspectiva han sido examinados con profundidad en el libro "Development as Freedom" (1999). Sen destaca el trabajo realizado en conjunto con Martha Nussbaum respecto a capacidades y calidad de vida (1993). El texto de Nussbaum (2001) "Women and Human Development. The Capabilities Approach" sintetiza su trabajo en esta perspectiva.

3 En la conclusión del capítulo sobre mercados, estado y oportunidad social de su libro Desarrollo y Libertad, Sen señala que los individuos viven y actúan en un mundo de instituciones. "Nuestras oportunidades y perspectivas dependen sobre todo de las instituciones que existen y de cómo funcionan. Las instituciones no sólo contribuyen a aumentar nuestra libertad, sino que su papel puede evaluarse de manera razonable a la luz de su contribución a aumentarla. La concepción del desarrollo como libertad permite evaluar sistemáticamente las instituciones". 
entrega de servicios sociales básicos, como educación, salud, redes de seguridad social, las cuales no necesariamente aparecen reflejadas en los ingresos reales de las personas.

El aumento o crecimiento de los ingresos de las personas probablemente expandirá las capacidades de las personas, con mayor énfasis en aquellas de ingresos más bajos. Sin embargo, desde el planteamiento de Sen, esto no puede ser considerado en sí mismo como el criterio final del desarrollo o del bienestar. Por ello, y siguiendo la perspectiva del autor, la pobreza debe concebirse como la privación de capacidades básicas y no sólo como la falta de ingresos. Asimismo, este enfoque no rechaza la idea razonable de que la falta de ingreso es una de las principales causas de la pobreza, ya que la falta de ingreso puede ser una importante razón por la que una persona esté privada de capacidades.

Pressman y Summerfield (2000) destacan lo apropiado del enfoque de Sen para analizar la pobreza utilizando el enfoque de las capacidades en oposición al ingreso como criterio único:

a) La pobreza puede identificarse de forma razonable con la privación de capacidades: el enfoque centra la atención en las privaciones que son intrínsecamente importantes a diferencia del bajo ingreso, que es sólo instrumentalmente importante.

b) Hay otros factores que influyen en la privación de capacidades, y por tanto en la pobreza real, además de la falta de ingreso. El ingreso no es el único instrumento que genera capacidades.

c) La relación instrumental entre la falta de ingreso y la falta de capacidades varía de unas comunidades a otras e incluso de unas familias a otras y de unos individuos a otros. La influencia del ingreso en las capacidades es contingente y condicional.

Además del vínculo entre capacidades y pobreza, Sen (1999) asocia el concepto de las capacidades al concepto de desarrollo. De este modo señala que se debe evaluar el desarrollo en términos de la expansión de las capacidades de las personas en vez de enfocarlo solamente como crecimiento económico. La combinación de capacidades y posibilidades concretas es lo específico del desarrollo y la libertad. Sen subraya que la evaluación de la libertad puede ser susceptible tanto a lo que hace una persona como a las alternativas que tiene; la libertad proporciona una perspectiva más amplia para juzgar la ventaja humana, y por medio de ella, evaluar el éxito social.
Este es el razonamiento básico que proporciona la base para considerar "el desarrollo como libertad". Desde la perspectiva de Sen, el proceso de participación debe ser entendido como una parte constitutiva de los fines del desarrollo en sí mismos. De este modo, la preocupación por la gobernabilidad de las instituciones no se debe solamente a que mejores instituciones aumenten nuestra habilidad para obtener fines ya dados por la teoría económica, sino a que desde las instituciones democráticas proviene la única manera de definir adecuadamente lo que pueden ser los fines económicos deseados. Las instituciones democráticas también ofrecen, en si mismas, la oportunidad de ejercitar una de las más importantes capacidades humanas, la capacidad de deliberar y de tomar decisiones.

La habilidad para tomar decisiones depende de las expectativas compartidas de un conjunto de personas acerca de cómo puede y deben proceder los intercambios y las discusiones de naturaleza pública y abierta. Cómo construir esas capacidades a través de reglas y organizaciones que generen y recompensen tales expectativas constituye uno de los desafíos más importantes. A pesar de colocar la deliberación en el centro de su concepto de desarrollo, Sen no analiza cómo los intercambios y discusiones públicos efectivos pueden encaminarse a crear instituciones fuertes.

Siguiendo esta perspectiva conceptual, es posible afirmar que las personas aumentan su bienestar cuando son capaces, por ejemplo, de leer, comer y votar. En este sentido, lo importante de saber leer se deriva de lo que se puede llegar a ser, de comer porque es necesario para la vida y la salud, y del votar porque se valora un determinado tipo de sistema político y una determinada forma de hacer política. El número de opciones que las personas tienen y la libertad de elección sobre estas opciones contribuyen al bienestar humano.

En su libro "Desarrollo como Libertad", Sen compara las tasas de supervivencia de hombres y mujeres en tres regiones del mundo: la población blanca y la afro-americana de Estados Unidos, la de China y la del estado de Kerala en India. Con estos datos Sen intenta destacar que, si bien el ingreso per capita de los afro-americanos de Estados Unidos es considerablemente menor que el de la población blanca del mismo país, los afro-americanos son mucho más ricos, desde el punto de vista de los ingresos, que los habitantes de China o de Kerala. Sin embargo, y a pesar de la notable diferencia de ingresos con los afro-americanos, los hombres de China y Kerala 
viven hasta edades más avanzadas. Respecto a las mujeres, las afro-americanas terminan teniendo un patrón de supervivencia similar a las de China, que son mucho más pobres, y muy inferior al patrón de las de Kerala, todavía más pobres.

Con esta evidencia, Sen desarrolla una argumentación central en torno a una libertad fundamental: la capacidad de sobrevivir. El argumento de Sen es que debe integrarse al ingreso y la renta la perspectiva de las libertades y capacidades, y por supuesto su cara opuesta, la carencia de capacidades y la falta de libertad. Es por ello que la pobreza debe ser vista no sólo como falta de ingresos sino también como carencia de capacidades básicas. En este mismo sentido es que Sen focaliza su trabajo en la importancia del desarrollo de las potencialidades humanas. Para el autor, la economía debe permitir el desarrollo de estas capacidades en las personas, incrementando las opciones vitales.

El enfoque de las capacidades ha influenciado de manera determinante muchos programas y políticas públicas. De acuerdo a Pressman y Summerfield (2000) algunos de los impactos de la utilización de este enfoque son los siguientes:

a) Cambios en la forma de concebir el desarrollo económico y la economía del bienestar. A partir de este enfoque se incorpora la perspectiva de desarrollar las capacidades de las personas. La idea básica es que es posible hablar de desarrollo cuando las personas son capaces de hacer más cosas y no sólo cuando estas son capaces de comprar más bienes o servicios.

b) Cambios en las formas de medir la pobreza y la incorporación de nuevos indicadores para la medición del desarrollo humano. Uno de los planteamientos básicos que incorpora Sen a estas mediciones es que el incremento de ingresos no implica necesariamente un aumento del bienestar, debido a que al interior de los hogares generalmente no hay estrategias de cooperación y distribución de los recursos.

c) Introducción de los criterios de género como parte integral de los procesos de desarrollo. A partir de evidencia empírica, Sen demuestra que los niveles de desarrollo afectan en forma distinta a hombres y mujeres debido a diferencias en el acceso a distintos tipos de servicios y alimentación.

La idea de que las personas son más desarrolladas cuando son capaces de hacer más cosas genera una serie de implicancias cuando se conciben e implementan programas sociales, específicamente en cuanto a objetivos y resultados esperados. Del mismo modo, la consideración de que el ingreso no constituye la única forma de medir el desarrollo demanda un serio tratamiento al diseñar intervenciones sociales asistenciales. Finalmente, el criterio de género como parte integral de los procesos de desarrollo implica un esfuerzo fundamental para entender y afinar intervenciones sociales que buscar avanzar en los distintos tipos de equidades.

\section{El enfoque del capital social}

La discusión en torno al capital social ha sido particularmente prolífica en los noventa y parte del 2000 si bien ha tendido a disminuir en los últimos anos. Una de las disputas en la abundante literatura de esta concepto gira en torno a la primera publicación sobre el tema ${ }^{4}$. Sin embargo, ya existe cierto consenso de que fue Lyda Hanifan, un joven educador del Estado de Virginia en Estados Unidos, quien usó por primera vez el concepto de capital social.

En un ensayo publicado en 1916, Hanifan destaca la importancia del compromiso comunitario y de las redes de solidaridad entre los ciudadanos para apoyar la democracia y el desarrollo. Luego de este trabajo inicial, es posible encontrar diversas publicaciones que retomaron desde distintas fuentes y perspectivas la temática ${ }^{5}$, sin embargo, el concepto aparece sólidamente en el debate académico con las publicaciones de los sociólogos Pierre Bourdieu, en Francia, James Coleman, en Estados Unidos, y con el cientista político norteamericano Robert Putnam.

Si bien estos autores señalan elementos comunes en la definición de capital social, existen algunas diferencias importantes. Bourdieu $(1980,1983)$ lo define como la acumulación de recursos reales o potenciales ligados a la posesión de una red duradera de relaciones más o menos institucionalizadas de conocimiento y reconocimiento mutuo o, en otras palabras, a la afiliación a un grupo. El trabajo de Bourdieu, sin embargo, está más centrado en el

4 Para un análisis riguroso del concepto de capital social se sugieren los textos de Woolcock (1998) y Portes (1998). También constituye un valioso aporte la introducción del texto de Putnam (2003) "El declive del capital social".

5 Al considerar la historia del concepto, estos autores son relevantes: L.J. Hanifan (1916, 1920), J. Jacobs (1961), G. Loury (1977), Pierre Bourdieu $(1980,1983)$, James Coleman $(1987,1988)$ y Robert Putnam $(1993,2000,2002)$. 
concepto de capital cultural, el cual ha servido de fuente importante para numerosos estudios antropológicos que tienen como foco la cultura.

Coleman $(1988,1990)$, por su parte, define el capital social desde una perspectiva estructural. Este autor señala que el capital social se manifiesta tanto en el individuo como en la colectividad. En el ámbito individual se manifiesta en los grados de integración social, redes a las que se pertenece y expectativas de reciprocidad. En el ámbito colectivo establece jerarquías legítimas que establecen normas que regulan las relaciones de acuerdo con un sentido de justicia. Coleman desarrolla estudios longitudinales en los que compara resultados académicos en colegios estatales y católicos. Este autor se concentra básicamente en los efectos en la familia y en las comunidades. A partir de sus estudios se destacan la complementariedad del capital humano y del capital social, en el contexto de los logros educacionales e inequidad social en adolescentes.

Coleman busca entender el capital social como recurso. Desde esta perspectiva, se destacan tres elementos centrales: a) las obligaciones y expectativas, b) los canales de información y c) las normas sociales. El trabajo de Coleman tiene similitudes con el de Bourdieu en cuanto a su preocupación compartida por la educación. Sin embargo también tiene diferencias importantes, por ejemplo en la relevancia que le otorga Bourdieu al tema del poder que se refleja en su estudio de los grupos de elite.

Sin embargo, es Robert Putnam (1993, 2000) quien coloca el concepto de capital social en el centro del debate académico y de política pública con su libro "Haciendo funcionar la Democracia". A diferencia de lo que señalan la mayoría de las lecturas en torno a este trabajo, el objetivo inicial de Putnam no fue analizar las diferencias de desarrollo entre el norte y el sur de Italia. La pregunta central que se planteó en su investigación de más de veinte años fue: ¿cuáles son las condiciones para crear instituciones representativas efectivas, responsables y fuertes?

Putnam identificó en el proceso de descentralización italiano iniciado en la década de los 70 una excelente oportunidad para estudiar sistemáticamente el nacimiento y desarrollo de una nueva institución ${ }^{6}$. En definitiva, Putnam intenta explorar los orígenes de un gobierno efectivo, presentando una evaluación comprensiva y comparativa del proceso descentralizador en cada una de las veinte regiones. Esto le permite indagar en los cambios a través del tiempo y hacer comparaciones entre las distintas regiones de las diferencias en desempeño institucional.

Los hallazgos de la investigación de Putnam en Italia se concentran en el vínculo entre desempeño institucional y comunidad cívica, lo que lo lleva a preguntarse por qué algunas regiones son más cívicas que otras. La respuesta a esta pregunta la encuentra en una argumentación histórica que gira en torno a la poderosa monarquía del sur de Italia y a un conjunto de repúblicas comunitarias del centro y norte del país. Considerando desde la época medieval hasta la unificación de Italia en el siglo XIX, Putnam encuentra el origen de las diferencias regionales en los patrones de participación cívica y solidaridad.

Putnam concluye que en el presente son estas tradiciones las que han tenido consecuencias decisivas para la calidad de vida pública y privada. De este modo, establece la comunidad cívica como su variable explicativa central, y es desde la cual acuña el concepto de capital social. Al trabajar el concepto de capital social desde la perspectiva de Putnam, son también tres los elementos centrales que se destacan: a) normas, b) redes, y c) confianza.

A partir del trabajo de Putnam, las publicaciones en torno al capital social alcanzan un crecimiento vertiginoso, aunque con ellas también aparecen las críticas. A continuación se mencionan algunas de ellas. Portes (1998) señala que el conjunto de procesos que alude el concepto de capital social no son nuevos y han sido estudiados bajo otros nombres en el pasado, en términos simples este concepto es sólo vino añejo en vasijas nuevas. A su vez, Portes y Landolt (2000) argumenta que muchas de las controversias del concepto de capital social tienen que ver con su aplicación a problemas que se encuentran en diferentes niveles de abstracción y su uso, en teoría, involucra distintas unidades de análisis. Molyneux (2002) enfatiza que los investigadores no han incorporado la

6 Reconociendo diferentes perspectivas en la vertiente neo-institucional, Putnam señala que hay dos elementos coincidentes en ellas. Primero, que las instituciones determinan la politica, y segundo, que las instituciones están determinadas por la historia. Las instituciones, afirma Putnam, influencian los resultados de la politica porque ellas forman las identidades, el poder y la estrategia de los actores, y las $i$ 
perspectiva de género en sus análisis de capital social. Finalmente, Fine (2001) señala que el capital social es una expresión de la colonización de la economía en las ciencias sociales manifestada en su tendencia a reducir fenómenos complejos. Este autor es particularmente crítico y escéptico respecto a la utilización de este concepto por parte de los organismos financieros internacionales.

Desde la perspectiva económica, un tema relevante en la literatura sobre capital social corresponde a las distinciones entre stock y flujo y entre los niveles existentes del recurso y su productividad. Para avanzar en la discusión del concepto es necesario profundizar este debate. El capital social puede ser entendido de manera simple como una categoría de varios tipos de activos sociales que establecen un conjunto de beneficios. Estos activos comprenden el llamado stock de capital social, mientras que los beneficios son los flujos. De este modo, la visión que se tenga de la naturaleza del stock y del flujo va influir no sólo en la manera en que puede ser entendido el capital social, sino también en las respuestas ante la posibilidad de invertir en él.

Para entender la distinción entre stock y productividad es útil hacer un paralelo con otros tipos de capital, por ejemplo el capital físico. Un gran stock de capital puede ser utilizado con una baja productividad y eficiencia, mientras que un pequeño stock puede ser utilizado con gran eficiencia. En el contexto del capital social estos temas son relevantes porque nos alertan acerca de las posibilidades de utilizar eficientemente los diferentes stocks de capital social con que se cuenta.

En los conceptos de capital social de Putnam (1993) y de Fukuyama ${ }^{\top}$ (1995), no aparece la diferencia entre stock y flujo, planteado en otros términos entre lo que es el capital social y lo que produce. Ambos autores asumen que altos niveles o stocks de capital social invariablemente resultarán en altos flujos de beneficios, mientras que un menor stock, del mismo modo, redundará en bajos beneficios. La conexión entre stock y flujos es directamente proporcional e invariable (Krishna, 2000).

El asumir la perspectiva conceptual de estos autores genera una serie de consecuencias en cuanto al tema de la inversión en intervenciones sociales en esta área. El stock de capital social no podría ser au- mentado en el corto plazo y un determinado stock produciría un flujo de beneficios específico e invariable. Precisamente esta visión determinista, y de algún modo pesimista del capital social, es la que provee uno de los más ricos debates en torno al concepto. En términos de inversión en capital social aparecen de inmediato las opciones de crear capital social y/o fortalecer los flujos de beneficios que este genera. La distinción conceptual entre stock y flujo es relevante por sus implicancias tanto para la investigación empírica como para la intervención en capital social.

El reconocimiento de la distinción entre stock y flujo es también útil para identificar la noción esencial de cultura que subyace en las definiciones de capital social de Putnam y Fukuyama. Implícitamente en el caso de Putnam y explícitamente en el caso de Fukuyama, ambos señalan culturas más o menos confiables, otorgando estas características a regiones enteras e incluso a países, desdibujando y minimizando las particularidades de comunidades o de regiones más específicas. Desde esta perspectiva crítica del concepto de capital social, e implícitamente de cultura, los índices agregados de capital social a escala nacional o regional pueden ser fácilmente criticados (Woolcock, 1998).

Woolcock (2001) resuelve el debate entre stock y flujo, definiendo al capital social como las normas y redes que facilitan la acción colectiva. Desde esta perspectiva, es importante señalar que cualquier definición de capital social se debe centrar más en sus fuentes $u$ orígenes que en sus consecuencias. Es decir, lo importante para definir capital social es preguntarse qué es, más que preguntarse qué hace o produce. Woolcock no utiliza en su definición de capital social la confianza, pues esta sería un resultado de repetidas interacciones sociales, de instituciones sociales creíbles, de la reputación. Este autor afirma que para avanzar en términos de claridad conceptual, tiene más sentido entender el capital social como una variable relacional (sociológica) que como una variable psicológica (individual) o política (institucional nacional).

Al considerar esta definición de capital social centrada en sus orígenes o fuentes, es necesario destacar su naturaleza multidimensional. Woolcock, atendiendo a desarrollos conceptuales

7 Fukuyama (1995) define capital social como una capacidad que surge de la predominancia de la confianza en una sociedad o en parte de ella. El problema de esta definición es que tanto el capital social como la confianza están histórica y culturalmente determinados. De este modo, en su libro establece que sociedades o países pueden tener o no tener este atributo. Por ejemplo, clasifica a China, Francia, Italia y Corea del Sur como sociedades familísticas, o a Alemania y Japón como sociedades de alta confianza. 
anteriores, ${ }^{8}$ subraya tres dimensiones del capital social. La primera corresponde a los lazos de unión o bonding (expresión utilizada en inglés) que refiere a relaciones de solidaridad y apoyo típicas de un capital social afectivo y compacto. Ejemplos de este tipo de capital son las relaciones de solidaridad y apoyo mutuo de los miembros de las familias, amistades y vecinos. Woolcock (2000) situando esta dimensión en contextos de pobreza, señala que alude a salir adelante o a "arreglárselas" gracias a "gente como yo".

La segunda dimensión corresponde a lazos más difusos y extendidos, es un tipo de capital social que tiende puentes o bridging. Esta dimensión hace alusión a relaciones entre personas de diferentes grupos pero que comparten características demográficas, por ejemplo edad o sexo; alude también a las relaciones sociales de amistad más lejanas, a socios y compañeros de trabajo. Este tipo de capital social tiende a ser fundamental en las organizaciones de voluntariado de convocatoria abierta y en las asociaciones formales e informales que buscan comercializar productos entre ellas.

Las dos dimensiones anteriores hacen referencia a relaciones sociales de carácter más horizontal. Sin embargo, Woolcock también identifica una dimensión vertical del capital social: la de vinculación o linking. Esta dimensión se refiere a las relaciones de las personas y organizaciones con las instancias de poder político y económico, a las relaciones sociales con las instituciones formales, como por ejemplo las autoridades de gobierno, de policía, las asociaciones empresariales. Este tipo de capital social tiende a ser fundamental en estrategias de intervención social en contextos de pobreza ya que permite establecer relaciones y alianzas entre los más pobres y las instancias públicas y privadas.

Este enfoque multidimensional del capital social subraya la necesaria complementariedad y dinamismo entre las tres dimensiones, las cuales son fundamentales de entender para generar estrategias de intervención social. En general, aunque no necesariamente en todos los casos, los grupos más pobres de la población poseen fuertes lazos de unión y relaciones que les permite tender puentes con otras organizaciones y grupos. Sin embargo, los pobres carecen de relaciones con las instituciones económicas y políticas que les permitan mejorar su situación social.

El cuadro 1, basado en la definición de capital social de Woolcock (2001), muestra los determinantes y los resultados del capital social. Este esquema ayuda a clarificar el debate entre stock y flujo, y contribuye en el avance hacia mediciones más sólidas de capital social.

CUADRO 1

CAPITAL SOCIAL: DETERMINANTES Y RESULTADOS

\begin{tabular}{|c|c|c|c|}
\hline \multirow[b]{2}{*}{$\begin{array}{l}\text { Ejemplos de determinantes } \\
\text { de Capital Social }\end{array}$} & \multicolumn{2}{|c|}{ Capital social } & \\
\hline & $\begin{array}{l}\text { Redes en las cuales opera } \\
\text { la confianza y reciprocidad }\end{array}$ & Características de las redes: & $\begin{array}{l}\text { Ejemplos de resultados } \\
\text { del capital social }\end{array}$ \\
\hline $\begin{array}{l}\text { Características Personales: } \\
\text { Edad } \\
\text { Sexo } \\
\text { Salud } \\
\text { Características Familiares: } \\
\text { Relación de pareja } \\
\text { Estado civil } \\
\text { Presencia de niños } \\
\text { Actitudes y valores } \\
\text { Tolerancia a la diversidad } \\
\text { Objetivos compartidos } \\
\text { Características del área donde } \\
\text { viven: } \\
\text { Rural / urbano } \\
\text { Nivel socioeconómico } \\
\text { Porcentaje de redes en la } \\
\text { localidad } \\
\text { Conocimiento de la localidad } \\
\text { Seguridad de la localidad }\end{array}$ & $\begin{array}{l}\text { Lazos de unión (Bonding): } \\
\text { Lazos informales: } \\
\text { Familia } \\
\text { Amigos } \\
\text { Vecinos } \\
\text { Compañeros de trabajo } \\
\text { Puentes (Bridging): } \\
\text { Relaciones Generalizadas: } \\
\text { Personas de la comunidad } \\
\text { Personas en general } \\
\text { Personas en grupos cívicos } \\
\text { Vinculación (Linking): } \\
\text { Relaciones Institucionales: } \\
\text { Relaciones con los sistemas } \\
\text { Institucionales } \\
\text { Lazos con el poder }\end{array}$ & $\begin{array}{l}\text { Tamaño y extensión: } \\
\text { Numero de lazos informales } \\
\text { Numero de vecinos conocidos } \\
\text { Numero de contactos en el } \\
\text { trabajo } \\
\text { Densidad y cercanía: } \\
\text { Miembros de la familia con los } \\
\text { cuales tiene cercanía } \\
\text { Amigos cercanos } \\
\text { Vecinos cercanos } \\
\text { Diversidad: } \\
\text { Diversidad étnica de los } \\
\text { amigos } \\
\text { Diversidad educacional de los } \\
\text { grupos a los cuales pertenece } \\
\text { Diversidad cultural de la } \\
\text { localidad }\end{array}$ & $\begin{array}{l}\text { Bienestar individual y familiar: } \\
\text { Capacidades para conectar } \\
\text { Capacidades para emprender } \\
\text { Bienestar Público: } \\
\text { Salud pública } \\
\text { Educación } \\
\text { Vida Cívica: } \\
\text { Voluntariado } \\
\text { Cooperación comunitaria } \\
\text { Vecindario / bienestar local: } \\
\text { Tolerancia a la diversidad } \\
\text { Disminución de la criminalidad } \\
\text { Bienestar político: } \\
\text { Participación democrática } \\
\text { Gobernabilidad } \\
\text { Bienestar Económico: } \\
\text { Crecimiento } \\
\text { Reducción de la inequidad }\end{array}$ \\
\hline
\end{tabular}

Fuente: el autor, tomando como base los trabajos de Stone (2001), Stone y Hughes (2002), y Woolcock (2001).

8 Para desarrollar estas dimensiones, Woolcock $(1998,2000,2001)$ y Woolcock y Narayan (2000) consideran los aportes conceptuales de Cooley (1909) en su trabajo respecto a los grupos primarios; a Granovetter $(1973,1985)$ en sus investigaciones respecto a los lazos fuertes y débiles y al arraigo; a Gittell y Vidal (1998) en su investigación respecto a la organización comunitaria y las formas de construir capital social como una estrategia de desarrollo; a Briggs (1999) en su trabajo de movilidad habitacional de grupos sociales; a Fox (1997) en su trabajo respecto a como construir capital social en comunidades rurales en Méjico, y a Heller (1996) en su investigación respecto a trabajadores industriales en la India. 
El énfasis de Woolcock no esta en la confianza y la cooperación como elementos centrales del capital social. Woolcock ejemplifica este aspecto señalando que el ámbito en el cual se extiende la confianza total o parcial está determinado, en muchas ocasiones, por un pequeño círculo de amigos, personas cercanas, organizaciones e instituciones. Al considerar esta perspectiva, se puede establecer no sólo en quién se puede confiar, sino también cuánto se puede confiar en determinada persona, grupo o institución. La tarea de construir capital social, por tanto, consistiría en extender esas expectativas previas de mutua confianza para producir mayores resultados en las comunidades.

En este sentido las expectativas juegan un rol central en las decisiones respecto al comportamiento. Las expectativas relativas a las posibles conductas de las personas y sus mismas expectativas influyen en el comportamiento de las comunidades. Cuando las diversas expectativas son convergentes y complementarias, el resultado es la cooperación. Si las expectativas son divergentes o disímiles, la cooperación se hace más difícil ${ }^{9}$.

Otro aporte relevante para el concepto de capital social que considera las expectativas es el de Krishna (2000). Este autor distingue capital social institucional y capital social relacional. En su propuesta, Krishna intenta integrar la visión racionalista y culturalista en ciencias sociales, señalando que estas dos dimensiones se refuerzan mutuamente y deben ser entendidas como un proceso. Sin embargo, Krishna deja sin contestar la pregunta respecto a cómo estas dos formas de capital interactúan a través del tiempo, elemento central para intentar invertir o intervenir desde esta perspectiva conceptual. Construir capital social, desde la visión de este autor, es equivalente a crear expectativas apropiadas. Sin embargo, no es sólo la cultura o las preconcepciones las que limitan las expectativas que conducen a más o menos confianza. Son también las instituciones, que reflejan el pasado y el presente de las condiciones estructurales de la sociedad, que actúan como limitantes o propiciadoras de la confianza.

En América Latina es importante destacar, entre muchas otras, las investigaciones de Fox (1997) en comunidades rurales de Méjico, Durston (1999, 2002) en comunidades campesinas de Guatemala y Chile, Bebbington (1998), Grootaert y Narayan (2000) en Bolivia, y Flores y Rello (2003) en Méjico y Centro América. En estas investigaciones se destaca el valor de las redes sociales, la participación ciudadana, la importancia de la cultura y los valores, los cuales son fundamentales de considerar al diseñar intervenciones sociales.

\section{El enfoque de las instituciones}

El tema de las instituciones sociales ha ganado importancia significativa en las últimas décadas en la teoría del desarrollo y en la creación de políticas y estrategias de intervención social dirigidas a combatir la pobreza. Sin embargo, el concepto de institución tiene múltiples interpretaciones, no sólo en las diferentes disciplinas de las ciencias sociales, sino también al interior de ellas. Dentro de las distintas corrientes, el neo institucionalismo económico ha ido ganando importante reconocimiento. Uno de sus precursores, el premio Nóbel de economía Douglass North (1990), señala que el desarrollo es el resultado del funcionamiento eficiente de las instituciones sociales. North (1986) define las instituciones como el conjunto de restricciones basadas en reglas y regulaciones, en la moral y la ética, y en las normas de conducta de una sociedad que dan forma a la conducta e interacción entre las personas. En términos simples, North señala que las instituciones son las reglas del juego de una sociedad.

En general, la economía neo-institucional puede ser entendida como un intento de incorporar la teoría de las instituciones en la economía. Intenta básicamente mostrar las condiciones bajo las cuales surgen las instituciones económicas particulares y los efectos de estas instituciones en el funcionamiento del sistema económico. Williamson (1994), quien da el nombre a la nueva economía institucional (Coase, 1998), afirma que la economía Neo-institucional atiende a dos premisas centrales en su génesis: las instituciones son importantes y los determinantes y consecuencias de las instituciones se pueden analizar con las herramientas de la teoría económica. Williamson (2000), señala tres aspectos relevantes de las instituciones. Primero, que son muy complejas y que todavía existe desconocimiento en torno a ellas; segundo, que en los últimos veinticinco años ha habido un progreso enorme en su estudio; $y$ finalmente, que mientras no haya una teoría unificada, es necesario aceptar el pluralismo teórico existente. Este autor también señala que los elementos cen-

9 Desde esta perspectiva es importante atender a las investigaciones desarrolladas por Elinor Ostrom (2000, 2003), especialmente a sus últimas publicaciones en torno a capital social, confianza y reciprocidad. 
trales asociados a la escuela neo-institucional son: a) los derechos de propiedad, entendidos como los derechos de un actor a usar activos valiosos; b) los costos de transacción, entendidos como el costo de oportunidad que implica para los actores establecer y mantener el control interno de los recursos (su papel es analizado como la conexión entre las instituciones y los costos de producción); c) los temas de información incompleta e incentivos.

El postulado básico de la perspectiva de North señala que las instituciones reducen la incertidumbre, minimizan los costos de transacción y crean incentivos para la exitosa cooperación entre los actores. El ejemplo al que regularmente hace referencia North es el de las reglas y normas utilizadas en un partido de fútbol. Son precisamente el conjunto de reglas que rigen el comportamiento de los jugadores en el campo de juego las que reducen la incertidumbre, permiten acuerdos básicos entre los actores y establecen incentivos para una cooperación exitosa. Siguiendo este ejemplo, North también enfatiza la obligatoriedad del cumplimiento de las leyes, es decir, el árbitro cuenta con reglas que le permiten expulsar a un jugador cuando éste no las sigue, y a su vez, el jugador acepta estas reglas. Finalmente, North señala que en un partido de fútbol no sólo existen reglas y normas formales sino que también hay un conjunto de reglas informales que pueden determinar la conducta de los jugadores.

Arrow, premio Nóbel de economía, señala respecto a esta perspectiva que la escuela neo-institucional es un movimiento dentro del paradigma o escuela neo-clásica (Williamson, 2000). El neo-institucionalismo se aleja de la escuela neo-clásica, pero no la abandona sino que la modifica y extiende. En este sentido, el neo-institucionalismo económico puede caracterizarse por los siguientes elementos: a) abandona la racionalidad instrumental, a la vez que plantea los límites de la racionalidad; b) acepta las premisas de la escasez y la competencia; c) emplea la teoría de precios como parte esencial del análisis de las instituciones; d) incorpora las ideas e ideologías dentro del análisis económico, modelando los procesos políticos como un factor central en el desempeño de las economías.

La economía neo-institucional, afirma Williamson, intenta responder nuevas preguntas: por qué las instituciones económicas emergen de una manera y no de otra. Si bien esta escuela emergente tiene una amplia fortaleza en el análisis teórico, aún señala un débil trabajo empírico. Una de sus características más relevantes es que tiene una perspectiva interdisciplinaria: derecho, antropología, sociología, ciencia política y otras disciplinas. Básicamente, desde la perspectiva económica neo-institucional, se postula que los individuos típicamente actúan con información incompleta y con modelos subjetivos.

Dentro de las diversas perspectivas del estudio de las instituciones, y también relacionados con el neoinstitucionalismo en ciencias sociales, se encuentran los estudios vinculados a la economía política de las instituciones sociales. Al interior de esta amplia perspectiva, también es posible encontrar diversos tipos. Sin embargo, se pueden clasificar bajo dos criterios: a) aquellos que estudian los efectos de las instituciones para explicar su manutención y estabilidad, y b) aquellos que estudian los mecanismos del cambio institucional.

Del mismo modo, complementando diferentes perspectivas conceptuales, es importante destacar que existen instituciones formales e informales, de tendencia privada y de tendencia pública, locales, nacionales y globales. Las instituciones sociales informales son aquellas que emergen y son mantenidas sin el apoyo explícito del Estado, mientras las instituciones formales son aquellas impuestas por la autoridad legal del Estado. Debido a las múltiples conexiones entre lo público y lo privado y a la imposibilidad de plantear en forma tajante el origen y función de las instituciones, se señala que es más útil hablar de instituciones de tendencia pública y de tendencia privada.

En términos conceptuales, un debate aún no resuelto corresponde a las definiciones de las instituciones en distintos niveles. Se señala que hay instituciones locales, nacionales o globales, sin atender claramente a sus posibles diferenciaciones conceptuales. También es importante atender a la diferenciación que realiza North (1990) respecto a instituciones y organizaciones. Para North, las organizaciones corresponden a los actores, mientras que las instituciones son las reglas del juego.

En términos generales se plantea que las instituciones formales e informales son importantes porque afectan el proceso de toma de decisiones y determinan la estructura de incentivos de una sociedad. De esta manera, se señala que son precisamente las instituciones políticas y económicas las que establecen los determinantes del desempeño económico. El funcionamiento eficiente y legítimo de las instituciones sociales se manifiesta generalmente en las relaciones sociales basadas en normas. 
Es por ello que se destaca la importancia de las estructuras legales como complemento fundamental en los proyectos de desarrollo. Sin embargo, si estas estructuras legales no son las adecuadas, pueden constituirse en los principales obstáculos en la implementación de proyectos. También se afirma que las instituciones son importantes debido a que las políticas públicas se formulan y operan desde instituciones y debido a que la gobernabilidad del sistema social depende de las instituciones.

Los autores del Nuevo Institucionalismo Económico señalan que la calidad de las ideas incorporadas en las instituciones es esencial para explicar las diferencias en las tasas de crecimiento. Hoff y Stiglitz (2001) señalan que la nueva teoría del crecimiento económico y el análisis organizacional moderno del crecimiento económico sitúan a la teoría del desarrollo en una comprensión compleja donde las instituciones tienen un papel fundamental en el desarrollo.

En América Latina es importante destacar la investigación de Stone, Levy y Paredes (1996). Estos investigadores analizan las interrelaciones y posibilidades de substitución entre las instituciones formales e informales. Específicamente analizan la industria de confección de ropa de Brasil y Chile con relación al contexto legal y las regulaciones para el inicio de los negocios.

El resultado de su análisis comparativo entre ambos países señala que si bien Chile posee instituciones formales más eficientes, Brasil posee instituciones informales que substituyen en forma también eficiente sus carencias. En este sentido, es importante mencionar que la investigación empírica de las interrelaciones entre instituciones formales e informales se encuentra en su desarrollo inicial. Finalmente, se destaca en la región la investigación del Banco Mundial en torno a instituciones locales en Bolivia (Grootaert y Narayan, 2000).

\section{Modelo analítico: el encuentro de los tres enfoques conceptuales en lo local}

Los enfoques de las capacidades, el capital social y las instituciones están relacionados con la ampliación del concepto de desarrollo. En este sentido, diversos autores han vinculado el desarrollo con las complementariedades entre capacidades e ins- tituciones o entre instituciones y capital social. Por ejemplo, en su planteamiento de expansión de las capacidades de los individuos, Sen le asigna un papel central a las instituciones.

Evans (2002a), considerando como punto de partida el enfoque de las capacidades de Sen, destaca la importancia que tienen las instituciones para favorecer el intercambio y la discusión pública ${ }^{10}$. Woolcock (2001) en su planteamiento del capital social destaca la importancia que tienen las instituciones para generar capital social. La propuesta de este documento es que los tres enfoques poseen múltiples posibilidades de complementación, y por tanto, mayores posibilidades potenciales de lograr desarrollo cuando son analizados en conjunto y en ámbitos locales.

En América Latina existe abundante literatura en torno al desarrollo local. Sin embargo, la definición de lo local continúa siendo ambigua e imprecisa. Acertadamente Boisier (1999) afirma que no hay respuestas convincentes en torno a la definición de lo local: "¿Qué es lo local?. ¿Se trata de una escala que habría que precisar en número de habitantes o en kilómetros cuadrados? ¿Supone un sistema de interacciones con una cierta autonomía? ¿Se refiere a una unidad político administrativa?". Desde una perspectiva antropológica, Appadurai (1997) afirma que lo local hace referencia a como se instalan (embedding) realidades de mayor escala en mundos concretos. A su vez, Appendini y Nuijten (2002) señalan que el contexto institucional local hace referencia al entorno institucional relevante para los hogares y las personas en espacios o áreas geográficas específicas.

Siguiendo a estos autores, lo local no alude necesariamente a territorios, ni tampoco a la estricta definición de un municipio como ámbito local. Se señala, por ejemplo, que un ministerio o una repartición que tiene una responsabilidad de coordinación nacional también tienen su expresión en un ámbito local. En efecto, en este tipo de organizaciones las personas se relacionan, comparten normas y valores, construyen identidades y se generan y recrean prácticas e instituciones sociales. Sin intentar saldar la discusión en torno al concepto, en este documento se entiende por lo local el ámbito analítico en el cual se expresan y es posible encontrar las instituciones, el capital social y las capacidades de los individuos.

10 Peter Evans destaca el concepto de desarrollo deliberativo y lo conecta con el de democracia deliberativa siguiendo los trabajos realizados por Bongham y Regh (1997), Elster (1998), y Fung y Wright (2001). 
El ámbito analítico de lo local incluye organizaciones de tendencia pública y privada. Se acentúa este aspecto difuso por la dificultad que envuelve categorizar a organizaciones e instituciones como sólo públicas o enteramente privadas. Además, porque se busca enfatizar la búsqueda permanente de complementariedades y sinergias (Evans, 1996a, 1996b) entre instituciones y organizaciones de origen público, privado y mixtas.

Es precisamente en esta definición de lo local donde es posible observar con más detalle la importancia de las instituciones informales y su interacción con las instituciones formales. En esta interacción muchas veces se sitúan los principales obstáculos o bien los principales facilitadores de los procesos de reforma y cambio institucional.

Además, esta definición de lo local presenta una necesaria visión balanceada del papel e importancia del estado central y de las instituciones y organizaciones que operan en el nivel territorial o micro (Serageldin y Grootaert, 2000). Lo local, desde esta visión, explicita la necesaria dependencia y posibilidades de articulación de los procesos, organizaciones e instituciones que operan y determinan la globalización económica, cultural y política con las realidades locales.

Otro elemento que subraya este enfoque analítico de lo local son sus posibilidades de aplicación a diferentes contextos, reconociendo las significativas diferencias entre realidades urbanas y rurales, pero también destacando los nuevos procesos de migración temporal de la ciudad al campo. En efecto, las economías más dinámicas de América Latina están mostrando importantes migraciones, no sólo del campo a la ciudad, sino también de trabajadores de las ciudades a la industria agrícola en épocas o para tareas específicas.

En este sentido, los enfoques tradicionales que sólo hacen énfasis en los territorios muestran sus debilidades al intentar responder a estos movimientos de población. En el modelo analítico propuesto (Cuadro 2) se identifican los tres distintos niveles que corresponden a los enfoques conceptuales desarrollados. Las capacidades se entienden al nivel de los individuos, el capital social en el ámbito grupal y comunitario y las instituciones en el ámbito comunitario y de la sociedad.

Es necesario entender y desarrollar las diferentes complementariedades entre los diversos niveles del modelo. Para ello es necesario recoger evidencias empíricas de formas de capital social alojadas en las instituciones locales que sirvan como ejemplos de buenas prácticas. También es necesario identificar tanto los mecanismos por los cuales estas experiencias resultaron ser exitosas como aquellos aspectos que todavía están pendientes.

El uso de indicadores para identificar, monitorear y establecer impactos positivos de intervenciones locales es fundamental. Los indicadores utilizados por Putnam $(2000,2001)$ y Narayan y Pritchett (1999) son un buen punto de partida para avanzar en esta línea.

Utilizando este modelo analítico resultaría interesante analizar las destacadas experiencias de intervención social de Villa el Salvador en Perú, el presupuesto participativo de Porto Alegre en Brasil o las ferias de consumo familiar en Venezuela. En estas tres realidades es posible especular que se dieron múltiples círculos virtuosos entre instituciones locales, capital social y construcción de capacidades.

CUADRO 2

MODELO ANALÍTICO

Integración de los Enfoques de las Capacidades (nivel individual), el Capital Social (grupal - comunitario) y las Instituciones (comunitario - societal).

\section{Sinergias y complementariedades}

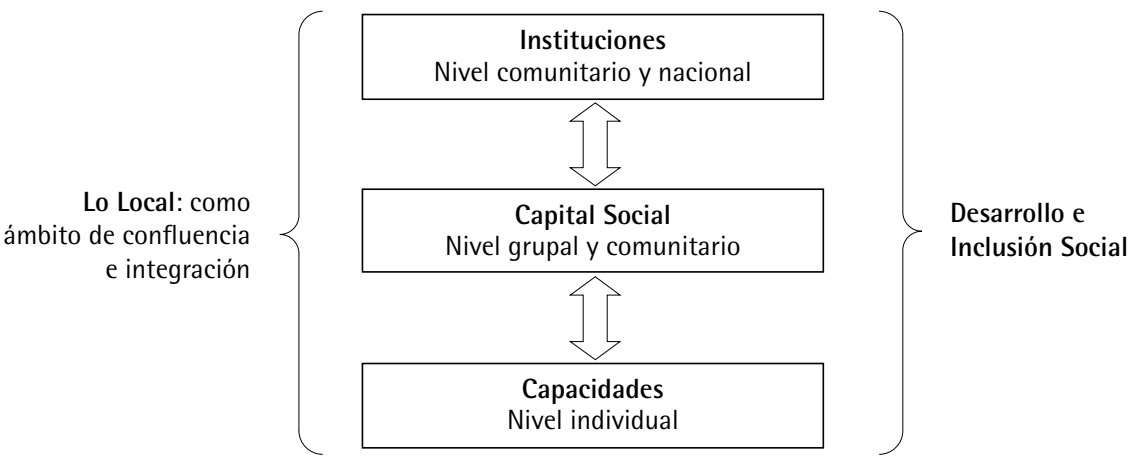


Habiendo colocado las coordenadas básicas de este modelo analítico, espacio privilegiado para analizar e intervenir en y con instituciones, capital social y capacidades, se enuncian un conjunto de sugerencias que recogen los aspectos conceptuales enunciados anteriormente. El mutuo refuerzo de la interacción entre las instituciones locales y las nacionales incrementa el stock de capital social. Las relaciones entre las instituciones formales e informales también necesitan ser consideradas. En el nivel local, las municipalidades y otras instituciones interactúan con una densa red de relaciones informales, estructuras y asociaciones de voluntarios. Estas interacciones definen las posibilidades y limitaciones de los individuos, de los hogares y de las actividades de los grupos.

La calidad de las instituciones es también importante. Las capacidades y efectividad de las instituciones en los niveles micro y macro y en las esferas formal e informal influencian los resultados de las intervenciones sociales publicas. Las instituciones necesitan valores pero también necesitan capacidades organizacionales, de administración y habilidades técnicas de comunicación para hacer funcionar estos valores.

En al ámbito local es posible observar relaciones sociales caracterizadas por densas redes sociales informales basadas en la confianza y reciprocidad de las relaciones cara a cara que constituyen recursos fundamentales para avanzar en procesos de desarrollo. El proceso de interacción y desarrollo del capital social y las instituciones es dinámico. El ejemplo citado frecuentemente en la literatura es el reemplazo gradual, durante un proceso de desarrollo exitoso, de las asociaciones y redes informales por estructuras administrativas formales y mecanismos de mercado impersonal. Además, si el patrón de desarrollo es apoyado y complementado por tribunales de justicia independientes que aseguren el cumplimiento de los contratos, capital social entendido como instituciones, todos los agentes económicos obtendrán ganancias.

El reconocimiento de las distintas dimensiones de capital social y sus complementariedades son necesarios para producir óptimos resultados de intervenciones sociales. Estos resultados pueden ser entendidos a través de la búsqueda de mejores indicadores sociales que den cuenta de experiencias de intervenciones sociales exitosas en las cuales se pueda demostrar los impactos obtenidos.

Estas intervenciones deben considerar todos los tipos de capital. En este sentido, el fortalecimiento y creación de capacidades individuales puede promover el capital social y mejorar el desempeño de las instituciones locales. En este sentido es importante identificar cuales son las instituciones formales e informales que incentivan la incorporación y participación de los pobres en los distintos mercados. Al respecto, el Informe de Desarrollo del Banco Mundial de 2002 señala que las preguntas relevantes son: ¿qué hacen las instituciones para promover el crecimiento y facilitar el acceso? ¿cómo se edifican estas instituciones? ¿de qué forma las instituciones pueden ayudar a las personas a utilizar mejor los activos que poseen y a multiplicarlos?

Sin embargo, no hay una única forma preestablecida para el desarrollo de las instituciones (World Bank, 2001). Por supuesto, lo básico es qué es lo que se puede hacer en el mundo real de nuestros días y no lo que debería hacerse en el mundo ideal. Para el desarrollo de las instituciones es necesario destacar la importancia de la historia, del liderazgo, las normas y la cultura.

Estas sugerencias e interrogantes tienen plena aplicabilidad en el contexto de las instituciones locales. Es decir, las estrategias de construcción de instituciones locales conectadas con los procesos de globalización pueden señalar muchas posibilidades de respuestas en términos de desarrollo y específicamente en términos de intervención social.

Scheneider et al (1997) señalan en su investigación empírica que el diseño de instituciones locales que entregan servicios sociales puede influenciar los niveles de capital social. Del mismo modo, la policía puede y de hecho afecta los niveles de capital social de las comunidades. El cambio en la estructura y composición de los centros de padres y apoderados de los colegios fortalece significativamente la participación de los padres en una amplia gama de actividades escolares, ayudando con ello a la construcción de capital social.

La existencia de instituciones que faciliten y apoyen las conductas cooperativas refuerza las expectativas de confianza y de reciprocidad. Sin estas instituciones que apoyen o fortalezcan este tipo de conductas podemos observar en la cultura fenómenos contrarios a la cooperación y la confianza. Al respecto, Cohen (2001) desarrolla la idea de intervenir en las instituciones como una manera de introducir el concepto de capital social dentro del poder político democrático.

Del mismo modo, Ostrom (2000) and Ostrom \& Ahn (2001) señalan que construir capital social a 
partir de intervenciones externas no es una tarea fácil y afirma que las instituciones regionales y nacionales afectan fuertemente el tipo de capital social disponible para acceder a procesos de desarrollo de largo plazo.

La participación es facilitada no sólo cuando los agentes externos son más receptivos a las opiniones de la comunidad, sino también cuando estos ayudan a las personas de la comunidad a construir instituciones, las cuales incluyen reglas y normas, estructuras, actitudes y desarrollo de capacidades. Estas instituciones son el soporte principal para apoyar las iniciativas de la comunidad en la identificación de problemas y alternativas de solución y en la implementación de aquellas seleccionadas. El sector público y el estado desempeñan un papel importante en la creación y fortalecimiento de las capacidades, el capital social y las instituciones, pero cómo deber ser entendido ese papel y cómo debería ser desarrollado es una pregunta que permanece sin contestar adecuadamente.

Los elementos mencionados anteriormente han sido enfatizados por diversos organismos internacionales de cooperación. El Banco Mundial ha destacado sostenidamente el papel e importancia de las instituciones locales para el desarrollo (World Bank, 1998). A su vez, Rodrik (1999) sugiere que puede ser útil pensar en instituciones políticas participativas como meta-instituciones que permitan obtener y agregar un conocimiento local y por tanto ayuden a construir mejores instituciones.

Del mismo modo, Fukuyuma (2003) señala que el agujero negro de la administración pública consiste en avanzar hacia soluciones locales para fortalecer la capacidad institucional. Agrega que proponer la capacidad institucional tiende a ser más un arte que una ciencia y que la mejor solución será la local, o al menos dependerá de una profunda comprensión de las condiciones locales.

\section{Consideraciones finales}

Este documento situó los conceptos de instituciones, capital social y capacidades en una primera aproximación a un modelo analítico. Si bien existe abundante literatura sobre los tres conceptos por separado, esta propuesta intenta integrarlos en un esfuerzo no sólo por entender las realidades locales de un modo más integral sino también como una alternativa efectiva para intervenir en ellas. De este modo se pretende contribuir a establecer las bases de un modelo de intervención social. Estos elementos conceptuales, y el modelo en sí mismo, se sustentan en el creciente consenso de que para producir más y mejor desarrollo es necesario considerar no solo recursos tradicionales como la tierra, el trabajo y el capital físico.

En el contexto de este modelo analítico de intervención social, la inversión social, entendida a través de su expresión en programas y proyectos sociales, debe considerar que la adquisición de capital humano y el establecimiento de capital físico necesitan ser complementados con relaciones sociales efectivas que se den dentro de un contexto institucional, sea este formal o informal.

Desde la perspectiva del modelo analítico propuesto, el análisis local institucional puede ayudar a identificar las capacidades y carencias de los sujetos que participan en las organizaciones y proyectos, los potenciales beneficios y dificultades del capital social en sus diferentes expresiones, y a la vez, modificar o crear instituciones sociales que aumenten los impactos de las intervenciones sociales.

Atendiendo a las premisas expuestas, se necesita mayor análisis e información respecto a mecanismos y metodologías utilizadas. Especialmente, es necesario analizar intervenciones sociales que hayan desarrollado círculos virtuosos entre las capacidades de los sujetos, el capital social y las instituciones en el ámbito local. A la vez, se necesita conocer e indagar en los errores y deficiencias que han tenido las intervenciones sociales, analizándolas desde la perspectiva del modelo propuesto.

Finalmente, se plantean algunas conclusiones para cada uno de los niveles del modelo propuesto.

En cuanto al capital social. Para generar relaciones sociales que favorezcan el desarrollo de programas y proyectos sociales se debe trascender la visión romántica de que el capital social puede solucionar todos los problemas y de que produce siempre resultados positivos. Sin lugar a dudas, este concepto ha permitido a docentes universitarios, formadores de políticas y profesionales de distintas disciplinas acceder a un interesante nivel de cooperación y dialogo. Pero es necesario confrontar y desarrollar las distintas dimensiones del capital social, especialmente aquellas interrelaciones que favorecen la inclusión de los pobres.

En cuanto a las instituciones. Para reducir la pobreza y otorgar facultades a los pobres, un aspecto central es encontrar las formas de crear sinergias entre las instituciones civiles y estatales y entre las instituciones formales e informales. Es importante atender al supuesto de que hay instituciones que favorecen la 
eficiencia y el desarrollo y otras, que por el contrario, pueden ser ineficientes y por tanto obstáculos importantes para el desarrollo.

En cuanto a las capacidades. Para profundizar la democracia y fortalecer la participación ciudadana una de las capacidades más importantes que es necesario desarrollar en los sujetos es la de deliberación. En este sentido, dar voz a los pobres es una acción que debe necesariamente ir complementada con la capacidad de tomar decisiones. En términos conceptuales, la perspectiva de las capacidades en el modelo propuesto es todavía amplia. Por lo tanto, es necesario seguir avanzando en una definición más rigurosa y restringida.

\section{Bibliografía}

APPADURAI, ARJUN. 1997. Modernity at Large: Cultural Dimensions of Globalization. Minnesota: University of Minnesota Press.

APPENDINI, KIRSTEN Y MONIQUE NUIJTEN. 2002. "El papel de las instituciones en contextos locales". Revista de la Cepal (76):71-88.

BEBBINGTON, ANTHONY. 1998. "Sustaining the Andes? Social Capital and Policies for Rural Regeneration in Bolivia". Mountain Research and Development 18 (2):173-418.

BEBBINGTON, ANTHONY. 1999. "Capitals and Capabilities: A Framework for Analizing Peasant Viability, Rural Livehoods and Poverty". World Development 27 (12):2021-2044.

BOISIER, SERGIO. 1999. "Desarrollo (local): ¿de qué estamos hablando?" Documento comisionado por la Cámara de Comercio de Manizales, Colombia. Santiago de Chile, 1999.

BONHAM, JAMES Y WILLIAM REHG. 1997. Deliberative Democracy. Cambridge, MA: MIT Press.

BOURDIEU, PIERRE. 1980. "Le capital social. Notes Provisoires" Actes de la Recherche en Sciences Sociales.

BOURDIEU, PIERRE. 1983. "Forms of Capital" En Handbook of Theory and Research for the Sociology of Education, edited by J. G. Richardson. New York: Greenwood Press.

BRIGGS, XAVIER DE SOUZA. 1998. "Brown Kids in White Suburbs: Housing Mobility and the Multiple Faces of Social Capital". Housing Policy Debate 9 (1): 177-221.

COASE, RONALD H. 1998. "The New Institutional Economics". The American Economic Review 88 (2):72-74.

COHEN, CATHY J. 2001. "Social Capital , Intervening Institutions, and Political Power". En Social Capital and Poor Communities, editado por S. Saegert, J. Phillip Thompson, and Mark R. Warren. New York: Rusell Sage Foundation.

COLEMAN, JAMES S. 1987. "Norms as Social Capital". En The Economic Approach Applied Outside the Field of Economics, editado por G. Radnitzky, and P. Bernholz. New York: Paragon House Publishers.

COLEMAN, JAMES S. 1988. "Social Capital in the Creation of Human Capital". American Journal of Sociology 94 (Complementado):S95-S120.

COLEMAN, JAMES S. 1990. Foundations of Social Theory. Cambridge, MA: Harvard University Press.

COOLEY, CHARLES HORTON. 1909. Social Organization: A Study of the Larger Mind. New York: Charles Scribner's Sons.

DURSTON, JOHN. 1999. "Construyendo Capital Social Comunitario". Revista de la Cepal 69:103-118.

DURSTON, JOHN. 2002. El Capital Social Campesino en la Gestión del Desarrollo Rural. Santiago, Chile: Comisión Económica para América Latina (CEPAL).

ELSTER, JON, ed. 1998. Deliberative Democracy. Cambridge: Cambridge University Press.

EVANS, PETER. 1996a. "Development Strategies across the Public-Private Divide". World Development 24 (6): 1033-1037.

EVANS, PETER. 1996b. "Government Action, Social Capital and Development: Reviewing the Evidence on Synergy". World Development 24 (6):1119-1132.

EVANS, PETER. 2002a. Beyond "Institutional Monocropping": Institutions, Capabilities, and Deliberative Development". En Visiting Scholar Russel Sage Foundation. New York.

FINE, BEN. 2001. Social Capital versus Social Theory: Political Economy and Social Science at the Turn of the Millennium. London: Routledge.

FLORES, MARGARITA Y FERNANDO RELLO. 2003. "Capital social: virtudes y limitaciones". En Capital social y reducción de la pobreza en América Latina y el Caribe: en busca de un nuevo paradigma, editado por Raúl Atria y Marcelo Siles. Santiago, Chile: Comisión Económica para América Latina (CEPAL) - Michigan State University.

FOX, JONATHAN. 1997. "How Does Civil Society Thicken? The political Construction of Social Capital in Rural Mexico". En State-Society Synergy: Government Action and Social Capital in Development, editado por P. Evans. Berkeley, California: UC Berkeley, International and Area Studies Publications.

FUKUYAMA, FRANCIS. 1995. Trust: The Social Virtues and the Creation of Prosperity. New York: The Free Press. 
FUKUYAMA, FRANCIS. 2003. "The Black Hole of Public Administration". IDB American Magazine, July, 2003.

FUNG, ARCHON Y ERICK OLIN WRIGHT. 2001. "Deepening Democracy: Innovations in Empowered Participatory Governance". Politics and Society 29 (1).

GITTELL, ROSS Y AVIS VIDAL. 1998. Community Organizing: Building Social Capital as a Development Strategy. Thousands Oaks, CA: Sage Publications.

GRANOVETTER, MARK. 1973. "The Strength of Weak Ties". American Journal of Sociology 78 (6):1360-1380.

GRANOVETTER, MARK. 1985. "Economic Action and Social Structure: The Problem of Embeddedness". American Journal of Sociology 91 (3):481-510.

GROOTAERT, CHRISTIAAN Y DEEPA NARAYAN. 2000. "Local Institutions, Poverty, and Household Welfare in Bolivia. Local Level Institutions". Working Paper 9. Washington, DC, Estados Unidos: Banco Mundial.

HANIFAN, L.J. 1916. "The Rural School Community Center". Annals of the American Academy of Political Science (67):130-138.

HANIFAN, L.J. 1920. The Community Center. Boston, MA: Silver, Burdett \& Company.

HELlER, PETER. 1996. "Social Capital as a Product of Class Mobilization and State Intervention: Industrial Workers in Kerala, India". World Development 24 (6): 1055-1071.

HOFF, KARLA AND JOSEPH STIGLITZ. 2001. "Modern Economic Theory and Development". En Frontiers of Development Economics, editado por G. Meier y J. Stiglitz. New York: Oxford University Press.

JACOBS, JANE. 1961. The Death and Life of Great American Cities. New York: Random House.

KRISHNA, ANIRUDH. 2000. "Creating and Harnessing Social Capital”. En Social Capital. A Multifaceted Perspective, editado por P. Dasgupta, and I. Serageldin. Washington, DC, Estados Unidos: Banco Mundial.

LOURY, GLENN. 1977. "A Dynamic Theory of Racial Income Differences". En Women, Minorities, and Employment Discrimination, editado por P. A. Wallace y A. LeMund. Lexington, MA: Lexington Books.

MOLINEUX, MAXINE. 2002." Gender and the Silences of Social Capital: Lessons from Latin America". Development and Change 33 (2):167-188.

MOORE, MARK H. 1995. Creating Public Value. Strategic Management in Government. MA: Harvard University Press.

NORTH, DOUGLASS C. 1986. "The New Institutional Economics". Journal of Institutional and Theoretical Economics 142:230-237.
NORTH, DOUGLASS C. 1990. Institutions, Institutional Change, and Economic Performance. New York: Cambridge University Press.

NUSSBAUM, MARTA C. 1995. "Human Capabilities, Female Human Beings". En Women, Culture, and Development: A Study of Human Capabilities, edited by M. C. Nussbaum, and Jonathan Glover. New York: Oxford University Press.

NUSSBAUM, MARTA C. 2001. Women and Human Development. The Capabilities Approach. Cambridge: Cambridge University Press.

OSTROM, ELINOR. 2000. "Social Capital: A Fad or a Fundamental Concept?" En Social Capital. A Multifaceted Perspective, editado por Dasgupta y Serageldin. Washington, DC: The World Bank.

OSTROM, ELINOR. 2003. "Toward a Behavioral Theory Linking Trust, Reciprocity, and Reputation”. En Trust and Reciprocity. Interdisciplinary Lessons from Experimental Research, Editado por Ostrom, E. New York: Rusell Sage Foundation.

OSTROM, ELINOR Y T.K. AHN. 2001. "A Social Science Perspective on Social Capital: Social Capital and Collective Action". Paper read at Social Capital: Interdisciplinary Perspectives, at Exeter, United Kingdom.

PORTES, ALEJANDRO. 1998. "Social Capital: Its Origins and Applications in Modern Sociology". Annual Review of Sociology 24:1-24.

PORTES, ALEJANDRO Y PATRICIA LANDOLT. 2000. "Social Capital: Promise and Pitfalls of its Role in Development". Journal of Latin American Studies 32:529-547.

PRESSMAN, STEVEN Y GALE SUMMERFIELD. 2000. "The Economic Contribution of Amartya Sen". Review of Political Economy 12 (1):89-113.

PUTNAM, ROBERT D. 2000. Bowling Alone. The Collapse and Revival of American Community. New York: Simon \& Schuster

PUTNAM, ROBERT D. 2001. "Social Capital Measurement and Consequences". En The Contribution of Human and Social Capital to Sustained Economic Growth and Well-Being. International Symposium Report, editado por J. F. Helliwell. Quebec: Human Resources Develeopment Canada (HRDC) and Organization for Economic Co-operation and Development (OECD).

PUTNAM, ROBERT D., ed. 2002. Democracies in Flux: The Evolution of Social Capital in Contemporary Society. Oxford: Oxford University Press.

PUTNAM, ROBERT D., ed. 2003. El Declive del Capital Social. Un Estudio Internacional sobre las Sociedades y el Sentido Comunitario. Barcelona: Galaxia Gutenberg. 
PUTNAM, ROBERT D., con Robert Leonardo y Rafaella Y. Nanetti. 1993. Making Democracy Work: Civic Traditions in Modern Italy. Princeton: Princeton University Press.

REPETTO, FABIAN. 2004. "Capacidad estatal: requisito necesario para una mejor política social en América Latina". Documentos de Trabajo. INDES - BID.

RODRIK, DANI. 1999. Making Openness Work. Maryland: Johns Hopkins University Press.

SCHNEIDER, MARK, PAUL TESKE, MELISSA MARSHALL, MICHAEL MINTRON et all. 1997. "Institutional Arrangements and the Creation of Social Capital: The Effects of Public School Choice". American Political Science Review 91 (1):82-93.

SEN, AMARTYA. 1984. Resources, Values and Development. Cambridge, MA: Harvard University Press.

SEN, AMARTYA. 1995. "The Concept of Development". En Handbook of Development Economics, edited by H. Chenery, and T. N. Srinivasan. Amsterdam: North-Holland.

SEN, AMARTYA. 1999. Development as Freedom. New York: Anchor Books.

SEN, AMARTYA Y MARTHA NUSSBAUM, ed. 1993. The Quality of Life. New York: Oxford University Press.

SERAGELDIN, ISMAIL AND CHRISTIAN GROOTAERT. 2000. "Defining Social Capital: An Integrating View”. En Social Capital. A Multifaceted Perspective, editado por Dasgupta y Serageldin. Washington, DC: The World Bank.

STONE, ANDREW, BRIAN LEVY, AND RICARDO PAREDES. 1996. "Transaction Cost and Economic Development. Public Institutions and Private Transactions: a Comparative Analysis of the Legal and Regulatory Environment for Business Transactions in Brazil and Chile". En Empirical Studies in Institutional Change, editado por Alston, Eggertsson, y North. New York: Cambridge University Press.
STONE, WENDY. 2001. "Measuring Social Capital. Towards a Theoretically Informed Measurement Framework for Researching Social Capital in Family and Community Life". Research Paper No. 24.Melbourne, Australia: Australian Institute of Family Studies.

STONE, WENDY AND JODY HUGHES. 2002. "Social Capital. Empirical Meaning and Measurement Validity". . Melbourne, Australia: Australian Institute of Family Studies.

WILLIAMSON, OLIVER E. 1994. "Transaction Cost Economics and Organization Theory". En The Handbook of Economic Sociology, edited by N. J. Smelser, and Richard Swedberg. Princeton, N.J.: Princeton University Press.

WILLIAMSON, OLIVER E. 2000. "The New Institutional Economics: Taking Stock, Looking Ahead". Journal of Economic Literature XXXVIII:595-613.

WOOLCOCK, MICHAEL. 1998. "Social Capital and Economic Development: Toward a Theoretical synthesis and Policy Framework". Theory and Society 27:151-208

WOOLCOCK, MICHAEL. 2000. "Managing Risk, Shocks, and Opportunity in Developing Economies: The Role of Social Capital". En Dimensions of Development, editado por G. Ranis. New Haven, CO: Yale Center for International and Area Studies.

WOOLCOCK, MICHAEL. 2001. "The Place of Social Capital in Understanding Social and Economic Outcomes". En The Contribution of Human and Social Capital to Sustained Economic Growth and Well-Being, editado por Helliwell. Quebec: Human Resources Develeopment Canada (HRDC) and Organization for Economic Cooperation and Development (OECD).

WOOLCOCK, MICHAEL, Y DEEPA NARAYAN. 2000. "Social Capital: Implications for Development Theory, Research, and Policy". World Bank Researcher Observer 15 (2):225-249.

WORLD BANK. 2001. World Development Report 2002: Building Institutions for Markets. New York: Oxford University Press.

FECHA DE RECEPCIÓN: septiembre 2005

FECHA DE ACEPTACIÓN: marzo 2006 